\title{
Impact of eating disorders on obstetric outcomes in a large clinical sample: a comparison with the HUNT Study
}

Eating Disorders and Obstetric outcomes

Trine Tetlie Eik-Nes ${ }^{1}{ }^{2 *}$, Julie Horn*3,4, Susanne Strohmaier ${ }^{5}$, Turid L. Holmen ${ }^{3}$, Nadia Micali ${ }^{6,7,8}$.

Sigrid Bjørnelv ${ }^{1,2}$

${ }^{1}$ Department of Mental Health, Norwegian University for Science and Technology, Trondheim, Norway N-7491, Norway

${ }^{2}$ Department of Mental Health and Addiction, Levanger Hospital, Nord-Trøndelag Hospital Trust, Levanger, Norway

${ }^{3}$ Department of Public Health and Nursing, Norwegian University for Science and Technology, Trondheim; Norway

${ }^{4}$ Department of Obstetrics and Gynecology, Levanger Hospital, Nord-Trøndelag Hospital Trust, Levanger, Norway

${ }^{5}$ Channing Division of Network Medicine, Brigham and Women's Hospital and Harvard Medical School, Boston, MA

${ }^{6}$ Department of Psychiatry, Faculty of Medicine, University of Geneva, Switzerland

${ }^{7}$ Division of Child and Adolescent Psychiatry, Department of Child and Adolescent Health, Geneva University Hospital, Geneva, Switzerland

${ }^{8} \mathrm{UCL}$ - Institute of Child Health, Child and Adolescent Mental Health Palliative care and Pediatrics Section, London, UK.

Word count (abstract): 244

Word count (manuscript): 4455

$1 *$ joint first authors 


\section{Acknowledgements}

Trine Tetlie Eik-Nes and Sigrid Bjørnelv had full access to all the data in the study and take responsibility for the integrity of the data and the accuracy of the data analysis. The NordTrøndelag Health Study (The HUNT Study) is a collaboration between HUNT Research Centre (Faculty of Medicine and Health Sciences, Norwegian University of Science and Technology NTNU), Nord-Trøndelag County Council, Central Norway Regional Health Authority, and the Norwegian Institute of Public Health. A special gratitude to Tove Haga, Hilde Kristin Vatterholm, Robert Iversen and Marlen Knutli who helped with data entry and preparation of the data set. This study would not have been possible without the thousands of women who participated in HUNT3 and the Hospital Registry at the Unit for Eating Disorders at Levanger Hospital.

Presentation information: This study was presented as an abstract at the Eating Disorders Research Society, Leipzig, Germany, September $15^{\text {th }}, 2017$.

Data sharing statement: The Nord-Trøndelag Health Study (HUNT) has invited persons aged 13 - 100 years to four surveys between 1994 and 2019. Comprehensive data from more than 125,000 persons having participated at least once and biological material from78,000 persons are collected. The data are stored in HUNT databank and biological material in HUNT biobank. HUNT Research Centre has been given concession to store and handle these data by the Norwegian Data Inspectorate. The key identification in the data base is the personal identification number given to all Norwegians at birth or immigration, whilst deidentified data are sent to researchers. Due to confidentiality HUNT Research Centre wants to limit storage of data outside HUNT databank, and we have restrictions for researchers for handling of HUNT data files. We have precise information on all data exported to different 
projects and there are no restrictions regarding data export given approval of applications to HUNT Research Centre. http://www.ntnu.edu/hunt/data

Availability of data from the Hospital Registry can be requested to the Data Access Committee (DAC) at the Health Trust of Nord-Trøndelag, Norway. 


\section{Abstract}

Objective: Current evidence from clinical studies suggests that having an active eating disorder (ED) during pregnancy is associated with unfavorable obstetric outcomes. However, the role of a lifetime diagnosis of ED is not fully understood. Variations in findings suggest a need for additional studies of maternal ED. This study aims to identify associations between a lifetime ED and obstetric outcomes.

Methods: Data from a hospital patient register and a population-based study (The HUNT Study) were linked to the Medical Birth Registry in Norway. Register based information of obstetric complications (preeclampsia, preterm birth, perinatal deaths, small for gestational age (SGA), large for gestational age (LGA), Caesarean sections and five-minute Apgar score) were acquired for 532 births of women with ED and 43,657 births of non-ED women.

Multivariable regression in generalized estimating equations was used to account for clusters within women as they contributed multiple births to the dataset.

Results: After adjusting for parity, maternal age, marital status and year of delivery, lifetime history of anorexia nervosa was associated with increased odds of having offspring who were SGA (Odds ratio (OR) 2.7, 95\% Confidence Interval (CI) 1.4-5.2). Women with a lifetime history of bulimia nervosa had higher odds of having a Caesarian section (OR 1.7 95\% CI 1.1-2.5). Women with EDNOS/sub-threshold ED had a higher likelihood of having a low Apgar score at 5 minutes (OR 3.1, 95\% CI 1.1-8.8).

Conclusion: Our study corroborates available evidence on the associations between maternal ED and adverse obstetric outcomes.

Keywords: Registry Studies, Eating Disorders, anorexia nervosa, bulimia nervosa, Eating Disorders Not Otherwise Specified, Birth Weight, Obstetric outcomes. 


\section{Introduction}

Eating disorders (EDs) including anorexia nervosa (AN) and bulimia nervosa (BN) are psychiatric disorders affecting about $8 \%$ of women during their reproductive age (Easter et al., 2013). It is not uncommon that women experience ED during pregnancy (Cynthia M.

Bulik et al., 2007; Easter et al., 2013; Watson et al., 2013), which is of concern as ED is associated with morbidity and poor nutritional status (N. Micali et al., 2012; Siega-Riz et al., 2008). Some women present with disordered eating (DE), i.e. ED symptoms lower in frequency and severity than a full-blown ED. Sub-threshold EDs are associated with a range of adverse mental and physical health consequences similar to those of full-blown ED (Field et al., 2012; Le Grange, Swanson, Crow, \& Merikangas, 2012). Regardless of frequency and severity, restrictive eating and purging behaviors may cause amenorrhea, irregular menstruation, and alteration of the normal hormonal balance (Crow, Thuras, Keel, \& Mitchell, 2002; Easter, Treasure, \& Micali, 2011; N. Micali et al., 2013), thereby severely compromising homeostatic balance and obstetric outcomes.

Studies may be biased because of the instability in ED phenotypes (Fairburn \& Harrison, 2003; Collier \& Treasure, 2004). Hence, a lifetime ED diagnosis may be a valid approach in defining ED phenotypes. Moreover, a focus on lifetime ED might have important implications for therapeutic interventions with pregnant women not caught by only focusing on current symptoms of EDs.

Numerous clinical studies have reported unfavorable obstetric outcomes in women with ED (Blais et al., 2000; Lacey \& Smith, 1987; Lemberg \& Phillips, 1989; Linna et al., 2014; Morgan, Lacey, \& Chung, 2006; Morgan, Lacey, \& Sedgwick, 1999; Morrill \& Nickols-Richardson, 2001; Pasternak Y, 2012; Sollid, Wisborg, Hjort, \& Secher, 2004; Stewart, Raskin, Garfinkel, MacDonald, \& Robinson, 1987). Some population-based studies, however, largely indicate no differences between women with EDs compared to controls in obstetric outcomes (Cynthia. M. Bulik et al., 2009; Ekéus, Lindberg, Lindblad, \& Hjern, 2006; N. Micali et al., 2012). This inconsistency may be due to low statistical power or less severe illness in population samples compared to clinical samples (Kimmel, Ferguson,

Zerwas, Bulik, \& Meltzer-Brody, 2016). Studies using data from clinical cohorts with linkage to medical birth registries with possible mediating factors are scarce, and findings may clarify existing inconsistencies. Therefore, we aim to examine the associations between a lifetime history of ED and obstetric outcomes in a large patient register compared to a populationbased study. Based on present evidence, we hypothesized that a lifetime diagnosis of EDs 
would be associated with adverse obstetric outcomes. In particular, we hypothesized that a lifetime diagnosis of AN would be associated with impaired fetal growth.

\section{Methods}

We obtained data from two sources in Norway. Patients from the patient registry constituted the exposed cohort and the third wave of the population-based HUNT Study (The Health Study of Nord-Trøndelag) was used as the referent after excluding any participants with symptoms of ED in HUNT3.

Data from the exposed women were retrieved from a local hospital patient register, consisting of patients diagnosed with ED at a specialized ED unit in Mid-Norway. Indication for admission to this ED unit, hence inclusion in this patient register takes account for prior unsuccessful outpatient treatment. All women who had been admitted to this unit, between January 2003-March 2015 and who had at least one birth recorded in the Medical Birth Registry of Norway (MBRN) in the time period from 1967-2015 were included in this study.

The HUNT Study is a population based study of health status in Nord-Trøndelag County, Norway, and comprises three large surveys (Krokstad et al., 2013). The referent cohort was selected from the third wave of HUNT (HUNT3), which was completed in 2008. The participation rate for women in HUNT3 was $58.7 \%$. The HUNT Study was considered to be the source population for the exposed women as both cohorts originated from the same area in Norway. Instead of introducing particular matching rules, we considered it beneficial to match the clinical cohort with a total HUNT3 cohort.

In total, 331 women with a lifetime history of ED and their births $(\mathrm{N}=644)$ were identified. In the referent cohort, we identified 21,510 women and their 49,735 births.

Both in the exposed and referent cohort, births were excluded if the birth was part of multiple births, the child weighed $<500$ grams, gestational length was $<22$ weeks and pregnancies with unlikely combinations of gestational age and birthweight (z-scores $\leq-4$ or $\geq 4$ ). We excluded multiple pregnancies, as these might have different patterns of fetal growth as well as different etiologic pathways for preeclampsia or preterm birth than singleton births.

There were insufficient numbers to study spontaneous abortion (defined as pregnancies with gestational length $<22$ weeks or birthweight $<500$ grams) as a separate outcome (Da Silva et al., 2016). In addition, we excluded births from the referent cohort where the mother had been identified as being at-risk of having an ED in HUNT3. To identify women at risk for ED in HUNT3, the Eating Disturbance Scale (EDS-5) and a shortened version of the Eating Attitude Test (Garner \& Garfinkel, 1979) (here referred to as the EAT-8) were used. EAT-8 included 
two factors, 'oral control' (EAT-A) and 'bulimia and food preoccupation' (EAT-B) (Bjomelv, Mykletun, \& Dahl, 2002; Rosenvinge et al., 2001). Women scoring above the $90^{\text {th }}$ centile (score $\geq 23$ ) on the EDS-5 and those who scored $\geq 3$ on the EAT- 8 and its subscales EAT-A, and $\geq 2$ for EAT-B were excluded. A thorough description of these screening instruments and derivation of cut-off scores for non-ED groups has been described earlier (Eik-Nes et al., 2015). A total of 6,084 births (12.2\%) were excluded in the referent cohort. Higher rates of small for gestational age (SGA) and possibly higher rates of perinatal mortality in women who have had bariatric surgery have been shown due to a likelihood of malnutrition or malabsorption (Johansson et al., 2015). Therefore, we additionally excluded births from the exposed cohort if a woman had bariatric surgery prior to pregnancy. Finally, we excluded births if a woman withdrew consent to participate, summing up to a total of 112 (17.4\%) excluded births from the exposed cohort. The final analytic study population (Figure 1) included 532 births from the exposed cohort and 43,651 births from the referent cohort.

\section{Exposure assessment}

The patient register at the ED Unit provided data on the exposed women's medical history, including ED diagnoses, ED behaviors and body mass index (BMI). Women were categorized into mutually exclusive diagnostic groups, reflecting ED on a continuum from restricting to bulimic (Collier \& Treasure, 2004). In order to define a lifetime history of ED, all medical records with ED diagnosis from first occurrence after the age of 18 to the end of the inclusion period were manually reviewed and reevaluated by a psychiatrist in the field of $\mathrm{ED}(\mathrm{SB})$ and the first author (TEN) until reaching consensus. ED diagnoses were assigned, according to the International Statistical Classification of Diseases and Related Health Problems, 10th revision (ICD-10) criteria (WHO, 1992). Women who did not meet diagnostic criteria of an ED, were categorized as having sub-threshold ED. The patients were grouped using broad criteria for $\mathrm{AN}$ and $\mathrm{BN}$ in analyses, with atypical forms combined with full disorders.

Furthermore, in line with the DSM-5 diagnostic criteria for ED which combines threshold ED and other specified feeding and eating disorders; we combined women with a lifetime history of EDNOS and sub-threshold ED (Diagnostic and statistical manual of mental disorders (5th ed.). 2013). In accordance with previous studies (Anderluh, Tchanturia, Rabe-Hesketh, Collier, \& Treasure, 2009; Eddy et al., 2008; N. Micali et al., 2007), we used a hierarchical approach for women who had more than one diagnosis over their lifetime (figure 2). As earlier studies of restricting type of AN have demonstrated a specific locus on chromosome 1 that has not been evident for a broader definition of AN, restricting type of AN may be a more 
homogeneous phenotype compared to other types of ED (Grice et al., 2002). Hence, in this study broad AN trumped broad BN, broad AN and BN trumped EDNOS and sub-threshold ED (figure 2). Additionally, as many women with an ED can display several symptoms of different ED diagnoses and thereby crossover from one ED diagnosis to another (Eddy et al., 2008), the exposed women were furthermore categorized according to specific ED behaviors (purging and restricting behavior) that exist across diagnoses. Women whose records included both restrictive and purging behaviors lifetime, where coded as lifetime purging, while those who only had records of restrictive behavior were assigned to the restrictive group (Figure 2). As we sought to study the effects of lifetime ED, analyses were carried out with all births irrespective of time of ED diagnosis.

\section{Outcome assessment}

In this study, identification of births and obstetric outcomes were extracted from the Medical Birth Registry of Norway (MBRN), which records virtually all births in Norway from 1967. (Irgens, 2000). Through compulsory notification, the MBRN collects detailed information on maternal and perinatal health, including complications during pregnancy and delivery. The birth notification practices remained unchanged from 1967 to 1998, and pregnancy complications were at that time described and reported in free text. As of 1999, maternal smoking habits and ultrasound based due dates were incorporated in a more detailed notification form (Irgens, 2002).

Preterm birth was defined as gestation length $<37$ weeks. We defined preeclampsia following international classification schemes as new onset of hypertension after 20 weeks of gestation together with proteinuria (Task Force on Hypertension in Pregnancy, 2013).

Z-scores of birthweights were calculated according to sex and gestational age, according to Norwegian standards (Skjaerven, Gjessing, \& Bakketeig, 2000). We chose to define SGA as z-score of birth weight adjusted for gestational age and sex below minus 2 (below the 3rd percentile) since this definition is more likely to distinguish growth restricted from constitutional small newborns (Figueras \& Gratacos, 2014). Similarly, LGA was defined as zscore above 2 (above 97th percentile). Caesarean section was dichotomized and included both elective and acute Caesarean sections. We dichotomized Apgar score at 5 minutes (Apgar 5) and low Apgar 5 was defined as <7. In 1967-1977, information on Apgar 5 was largely missing (98\%) from the MBRN. Later, between 1989 and 2015, Apgar 5 scores were reported in most births with only $1 \%$ missing. We defined perinatal mortality as stillbirth or death
Commented [NM1]: I am not sure this justifies the sentence that follows, can you chekc how we justified the hierarchy in our lifetime ED paper in our BMC medicine paper? I woudl respond something like we apologize for the lack of clarity, we used a hierarchial diagnosis with AN trumping BN, and $\mathrm{BN}$ trumping EDNOS, this is base don previous literature (cite treasure and collier and my own) and on disorder severity, given that $\mathrm{AN}$ is a more life-threatening disorder 
within seven days of birth. In Norway, two methods of estimation of gestational age are made accessible in the MBRN: (1) last menstrual period (LMP) (recorded from 1967) and (2) expected date of delivery according to ultrasound measurements (recorded from 1999) (Tunon, Eik-Nes, \& Grottum, 1996a). Analysis on SGA, LGA and preterm birth included analysis with both sources of estimation of gestational age and secondary analysis were restricted to pregnancies with ultrasound estimations.

\section{Covariates}

A priori confounders, based on previous studies (Kimmel et al., 2016) and likely to influence obstetric outcomes were included in the multivariate models. Maternal age, year of delivery, parity and marital status were collected from the MBRN. We entered maternal age at delivery as a continuous variable in the models. Significant advances in neonatal and perinatal care were in Norway gradually implemented during the 1980s (Bakketeig, 1984; WHO, 2006), hence, year of delivery was added as a binary variable with two time periods: 1967-1989 and 1990-2015. Parity (number of births) was categorized as: one, two, and 3 or more births. We added marital status as a binary variable in the models: divorced/single/widow or married/cohabitant/partner. Smoking was assessed with a binary answer (yes/no) during the first, second or last trimester. Information on smoking in any of the trimesters was considered as missing if a woman did not consent to provide data on smoking during pregnancy. Data on smoking was largely missing with only $8,375(19.2 \%)$ of the births of the total sample with complete data on smoking. As BMI was not routinely collected at the first antenatal visit in the MBRN before 2007, data on BMI was largely missing. However, we collected information on prepregnancy BMI in a sub-set of the study population to examine the role of pre-pregnancy BMI on the association between ED and obstetric outcomes. As the MBRN only had recorded pre-pregnancy BMI in 57 births for the exposed cohort (10.7\%), we manually obtained data on pre-pregnancy BMI from birth records in Mid-Norway, leaving a total of 103 (19.4\%) births with data on BMI pre-pregnancy (AN: $n=43),(B N: n=49)$, (EDNOS and sub-threshold ED: $n=11)$. In the referent cohort, pre-pregnancy BMI was recorded in 446 births (1.0\%) in the MBRN. Hence, as a proxy for BMI pre-pregnancy, we collected objectively measured BMI that was obtained within 2 years from the birth of the child in the referent cohort, leaving 972 births $(2.2 \%)$ with available data on BMI pre-pregnancy. In HUNT3, height (to the nearest centimeter) and weight (to the nearest half kilogram) were measured with the participants wearing light clothes and no shoes (Krokstad et al., 2013). We calculated BMI as weight divided by the squared value of height. 


\section{STATISTICAL ANALYSES}

We used multivariable regression in generalized estimating equations (GEE) to account for clusters within women as they contributed multiple births to the dataset, using STATA version 15 ("StataCorp," 2017). We specified an exchangeable correlation structure and used the logit link function to estimate odds ratios (ORs) and 95\% confidence intervals (CIs) of having experienced adverse obstetric outcomes comparing women with a lifetime history of different types of EDs to women with no ED. To investigate differences in continuous variables, we again specified an exchangeable correlation structure but used the identity link function. Analyses were adjusted for parity, marital status, maternal age, and year of delivery. The significance threshold was set at $\mathrm{p}<0.05$.

\section{Secondary analyses}

For mother/child pairs with available information on smoking during pregnancy (exposed $\mathrm{n}=322$, unexposed $=8,054$ ), we repeated the main analyses and examined how the much adding another confounder - smoking - would influence the results for the different types of ED. Furthermore, as ultrasound estimates of gestational age are considered to be more precise (Dietz et al., 2007; Tunon, Eik-Nes, \& Grottum, 1996b), we conducted sensitivity analyses on the outcomes SGA, LGA and preterm birth, restricting to ultrasound dated pregnancies. In all these secondary analyses, we considered the ED diagnosis analysis the more important analysis and did not want to distract from that with additional results of the ED behaviors. Due to low power, we were unable to repeat the regression models using mother/child pairs with information on pre-pregnancy BMI. Hence, we were unable to study possible mediation effects of pre-pregnancy BMI.

The Regional Ethical Committee of Mid-Norway granted ethical approval for the study. An exemption of consent was given, meaning women from the exposed cohort could withdraw from the study by actively not consenting to participate. Furthermore, an exemption of consent was also obtained to collect data from births $(\mathrm{N}=17)$ whose mothers in the exposed group had deceased $(\mathrm{N}=9)$. Participation in the HUNT3 survey and the present study was voluntary and based on written informed consent. The Norwegian Data Inspectorate, The Regional Committee for Ethics in Medical Research in Central Norway (REC Central) and the Norwegian Directorate of Health, approved the HUNT surveys.

\section{RESULTS}




\section{Main findings}

In the exposed group, we included 272 women who had given birth with a lifetime history of ED and their births $(\mathrm{N}=532)$. In the referent cohort, we included 19,049 women who contributed with 43,651 births. Mothers with a lifetime history of broad AN, broad BN, EDNOS and sub-threshold ED had 244, 226 and 62 births, respectively. Furthermore, women with restrictive ( $n=63)$ and purging behaviors $(n=196)$ contributed with 124 and 381 births, respectively. Table 1 shows number of pregnancies with mothers with lifetime ED behaviors across lifetime ED diagnoses.

Demographic characteristics can be found in Table 2. No differences in maternal age at delivery were found between the exposure groups. Women from the exposed cohort were less likely to be living with a partner than women without ED. The exposed women did not largely differ from the referent group regarding smoking. Women with $\mathrm{AN}$ and $\mathrm{BN}$ had lower pre-pregnancy BMI compared to women in the referent group.

Results from the GEE multivariable logistic regression models showing associations between different types of ED and obstetric outcomes are given in Table 3. After adjusting for parity, maternal age, marital status and year of delivery, women with a lifetime history of AN had higher odds of having offspring who were SGA (OR 2.7, 95\% CI 1.4-5.1). Women with EDNOS and sub-threshold ED had higher odds of having an offspring with a low Apgar 5 score compared to women from the referent group (OR 3.2, 95\% CI 1.1-8.9). Women with a lifetime history of BN had higher odds of having a Caesarian section (OR 1.7, 95\% CI 1.1-

2.5). Due to low power, regression models on perinatal mortality were not performed. Nevertheless, two children $(0.8 \%)$ died in stillbirth or within seven days of birth in the exposed cohort (both children of women with a lifetime history of AN). In the referent cohort, $508(1.2 \%)$ perinatal deaths were registered.

Results from the GEE multivariable logistic regression models showing associations between ED behaviors and obstetric outcomes are given in Table 4. Women with a lifetime history of purging had higher odds of having offspring who were SGA (OR 2.2, 95\% CI 1.33.8). No differences between the cohorts on other obstetric outcomes were found in these analyses.

\section{Secondary analyses}

General descriptive statistics of the subsample with and without available information on smoking during pregnancy can be found Supplemental Table 1. Results on associations 
between lifetime history of ED types and obstetric outcomes for the subset with available smoking information are given in Supplemental Table 2. Generally, results using this subsample are comparable to the results using the main sample in terms of direction and largely also in terms of magnitude. Additional adjustment for smoking did not substantially change the observed estimates.

In the sensitivity analyses restricting to ultrasound dated pregnancies, women with a lifetime history of AN had higher odds of having an offspring who were SGA (OR 5.1, 95\% CI 2.6-10.2) compared to using both LMP dated and ultrasound dated pregnancies SGA (OR 2.7, 95\% CI 1.4-5.1) (Supplemental Table 3). In these analyses, women with a history of purging also had higher odds of having an offspring who were SGA (OR 4.8, 95\% CI 2.68.8). Due to low power, we were not able to perform regression models on obstetric outcomes among those with available information on pre-pregnancy BMI. However, in this sub-set, women with AN and BN had lower pre-pregnancy BMI compared to women in the referent group. Women with EDNOS and sub-threshold ED had a higher pre-pregnancy BMI compared to the referent cohort (Table 1).

We found no difference between those who withdrew ( $n=44)(A N: n=22),(B N$ : $\mathrm{n}=17)$, (EDNOS and sub-threshold ED: $\mathrm{n}=5)$ and those who participated $(\mathrm{n}=272)$ concerning smoking during pregnancy, maternal age or marital status.

\section{Discussion}

It has previously been shown that maternal EDs are associated with a range of adverse obstetric outcomes (Kimmel et al., 2016). In this study, we used a large clinical sample and a general population sample with linkage to a national birth registry to study adverse obstetric outcomes in women with a lifetime history of ED. We found that different maternal EDs were associated with increased odds of SGA, Caesarian sections and low Apgar score at 5 minutes, which largely corroborate previous findings. Adjustment for the included confounders did not change the main results.

Our finding that women with a history of AN had increased odds of having offspring who were SGA is consistent with some (Koubaa, Hallstrom, Lindholm, \& Hirschberg, 2005; Linna et al., 2014; N. Micali, Stemann Larsen, Strandberg-Larsen, \& Nybo Andersen, 2016; Sollid et al., 2004) but not all previous studies (Cynthia. M. Bulik et al., 2009; Ekéus et al., 2006; N. Micali et al., 2012). In contrast to our results, Cynthia. M. Bulik et al. (2009) found no association between maternal self-reported AN and SGA in a large Norwegian study population $(\mathrm{MoBa})$. Although our study population might overlap to a certain degree with the 
MoBa study population, the conflicting results between the studies might be explained by the lower severity of illness in the MoBa study. In particular, women with EDs might be less likely to participate in general population studies. For example, only 28 women (8.5\%) with ED from the local patient registry in our study participated in HUNT3.

In agreement to some (Cynthia. M. Bulik et al., 2009; Eagles, Lee, Raja, Millar, \& Bhattacharya, 2012; Ekéus et al., 2006; Koubaa et al., 2005; N. Micali et al., 2016) but not all (Linna et al., 2014; Sollid et al., 2004) previous studies, we found no differences between the cohorts with respect to giving birth preterm. Higher risk of preterm birth among women with EDs in some previous clinical studies might have been driven by women who had an active ED at conception (Linna et al., 2014; Sollid et al., 2004). The inconsistency in findings between studies may be due to methodological differences such as low statistical power or different classification of EDs.

In the sensitivity analyses restricting to ultrasound dated pregnancies, we found higher odds of having an offspring who were SGA compared to our main analysis. Generally, using LMP to estimate gestational age based is considered less accurate compared to ultrasound dating as it tends to classify more deliveries as preterm (Rappazzo, Lobdell, Messer, Poole, \& Daniels, 2017; Tunon et al., 1996b). However, our sensitivity analyses may be explained by factors that reduce fetal size which may increase the risk of preterm delivery when gestational age is based on ultrasound measurements (Henriksen, Wilcox, Hedegaard, \& Secher, 1995). Hence, potential misclassification of gestational age in assessment of underweight women should be considered, as lack of fat tissue may lead to a lack of estrogens and thereby disturb the menstrual cycle (Reid \& Van Vugt, 1987).

EDNOS has been given less attention in previous literature (Pincus, Davis, \& McQueen, 1999), even though it often is the most common diagnosis in clinical samples (Fairburn \& Bohn, 2005). We found that lifetime history of EDNOS, but not lifetime history of other EDs, was associated with a higher likelihood of having a baby with a low Apgar 5 score. This was unexpected given that EDNOS is considered to be less severe than full-blown EDs, however common comorbid psychopathology in women with EDNOS (Le Grange et al., 2012) might contribute to the observed association. However, the heterogeneity among individuals with EDNOS comprises a spectrum of severity and our results should therefore be interpreted with caution.

Women with a history of $\mathrm{BN}$ and those with a history of purging were more likely to have a Cesarean section compared to the referent group, in disagreement with other studies where no associations between maternal ED and Caesarian sections have been found (C. M. 
Bulik et al., 1999; Franko et al., 2001). This is particularly worrying, as Cesarean section carries risks for both the infant and the mother (Hager et al., 2004). Moreover, we found that women with a lifetime history of purging had higher odds of having offspring who were SGA. Hence, women with a history of purging may be at increased risk of having obstetric complications, suggesting that women with lifetime history of ED should be asked about current and previous purging behaviors when pregnant.

\section{Strengths and limitations}

This study has several strengths. The ED diagnoses were based on careful selection of cases and controls of clinical intake diagnoses, and information on continuation of ED behaviors across diagnostic groups, which was quality assured by ED specialists. We were also able to take into account the role of several covariates relevant to the outcomes.

Most studies on maternal EDs have been limited to variables that are recorded in national or hospital registries, opposed to our study where we used medical records with linkage to a national birth registry encompassing practically all births in Norway since 1967, ensuring thorough assessment and quality assurance. Misclassification of the outcomes is likely to have been non-differential. Another strength of the study was our ability to combine a clinical sample with a population-based one, in addition to our ability to include women with sub-threshold ED and EDNOS.

Some limitations have to be taken into account. Due to insufficient power we were unable to compare lifetime ED in remission with active EDs, and thus not able to compare chronic lifetime diagnoses with participants in recovery. Accurate data on length of illness and time since diagnosis, and whether or not patients had diagnoses that offset before gestation across their pregnancies was not available and hence not addressed in our analyses. However, the medical records showed that the mother had an active ED in $18.8 \%$ of the total number of pregnancies in the exposed cohort. More, indication for admission at this unit for ED take account of prior unsuccessful outpatient treatment and need for intensive treatment due to high severity of ED symptoms, inferring that many of the women had been treated for ED in primary or tertiary care earlier. Moreover, we were unable to adjust for socioeconomic status, however socioeconomic inequalities in health in Norway are less compared to other European countries (Mackenbach et al., 2008). Furthermore, the MoBa study showed that adjustment for income and education largely did not change the results in the association between ED and obstetric outcomes (Cynthia. M. Bulik et al., 2009). Likewise, we were unable to adjust for smoking in our main analyses due to a substantial amount of missing data. 
However, a sensitivity analysis restricted to women with available information on smoking showed no substantial change of the observed estimates after adjustment for smoking. Also, pre-pregnancy BMI may have mediated the associations in this study. Due to a large amount of missing data, we found that possible mediation effects would be hard to interpret. We could only identify women who were assessed or treated in Mid-Norway. It is thus uncertain if the results found in this study are generalizable to other women with ED. However, it is unlikely that these women differ from women in other clinical cohorts of ED in Norway as this Unit for ED treats patients from all over the country. Unmeasured confounding may have influenced our results, including changes in maternal and perinatal care in Norway during the study period. A substantial part (13.8\%) of the included sample withdrew their consent to participate, thus consent bias may be present. Identification of women with ED can be problematic as many of these experience a great deal of shame (N. Micali, \& Schmidt, U, 2007), which may have led some to withdraw their consent. However, women who withdrew consent did not largely differ from those who participated. Small sample sizes in outcomes and diagnostic groups might account for false negatives. Similarly, chance might explain some of our findings.

\section{CONCLUSION}

Our study corroborates available evidence on the association of maternal ED and obstetric complications. Specifically, our findings shed light on obstetric outcomes across ED diagnosis, including sub-threshold ED and EDNOS, which have been largely under researched. Also, our results suggest that specifically purging, which exist across ED diagnosis, are associated with increased odds of having obstetric complications. Thus, our findings should have implications for both obstetric and primary care, including awareness about the risk of adverse obstetric outcomes for women with an active ED or a lifetime history of ED. Specialized mental health providers who treat women with ED should work together with obstetrical providers, as well as general practitioners to improve the care for women with ED. Larger clinical studies are further needed to assess the risk of obstetric complications in women with lifetime and active ED to better understand risk mechanisms. 


\section{References}

Anderluh, M., Tchanturia, K., Rabe-Hesketh, S., Collier, D., \& Treasure, J. (2009). Lifetime course of eating disorders: design and validity testing of a new strategy to define the eating disorders phenotype. Psychol Med, 39(1), 105-114. doi:10.1017/s0033291708003292

Bakketeig, L. S. (1984). Perinatal omsorg $i$ Norge: helsearbeid blant svangre og fødende kvinner samt nyf $\phi$ dte barn. Oslo: Universitetsforlaget.

Bjomelv, S., Mykletun, A., \& Dahl, A. A. (2002). The influence of definitions on the prevalence of eating problems in an adolescent population. Eat Weight Disord, 7(4), 284-292.

Blais, M. A., Becker, A. E., Burwell, R. A., Flores, A. T., Nussbaum, K. M., Greenwood, D. N., ... Herzog, D. B. (2000). Pregnancy: outcome and impact on symptomatology in a cohort of eating-disordered women. Int J Eat Disord, 27(2), 140-149. doi:10.1002/(SICI)1098-108X(200003)27:2<140::AID-EAT2>3.0.CO;2-E [pii]

Bulik, C. M., Sullivan, P. F., Fear, J. L., Pickering, A., Dawn, A., \& McCullin, M. (1999). Fertility and reproduction in women with anorexia nervosa: a controlled study. J Clin Psychiatry, 60(2), 130-135; quiz 135-137.

Bulik, C. M., Von Holle, A., Hamer, R., Knoph Berg, C., Torgersen, L., Magnus, P., . . . Reichborn-Kjennerud. (2007). Patterns of remission, continuation and incidence of broadly defined eating disorders during early pregnancy in the Norwegian Mother and Child Cohort Study (MoBa). Psychol Med, 37(08), 1109-1118. doi:doi:10.1017/S0033291707000724

Bulik, C. M., Von Holle, A., Siega-Riz, A. M., Torgersen, L., Lie, K. K., Hamer, R. M., . . Reichborn-Kjennerud, T. (2009). Birth outcomes in women with eating disorders in the Norwegian Mother and Child cohort study (MoBa). Int J Eat Disord, 42(1), 9-18. doi:10.1002/eat.20578

Collier, D. A., \& Treasure, J. L. (2004). The aetiology of eating disorders. Br J Psychiatry, 185, 363-365. doi:10.1192/bjp.185.5.363

Crow, S. J., Thuras, P., Keel, P. K., \& Mitchell, J. E. (2002). Long-term menstrual and reproductive function in patients with bulimia nervosa. Am J Psychiatry, 159(6), 10481050.

Da Silva, F. T., Gonik, B., McMillan, M., Keech, C., Dellicour, S., Bhange, S., . . The Brighton Collaboration Stillbirth Working, G. (2016). Stillbirth: Case definition and guidelines for data collection, analysis, and presentation of maternal immunization safety data(). Vaccine, 34(49), 6057-6068. doi:10.1016/j.vaccine.2016.03.044

Diagnostic and statistical manual of mental disorders (5th ed.). (2013). American Psychiatric Association. Washington, DC.

Dietz, P. M., England, L. J., Callaghan, W. M., Pearl, M., Wier, M. L., \& Kharrazi, M. (2007). A comparison of LMP-based and ultrasound-based estimates of gestational age using linked California livebirth and prenatal screening records. Paediatr Perinat Epidemiol, 21 Suppl 2, 62-71. doi:10.1111/j.1365-3016.2007.00862.x

Eagles, J. M., Lee, A. J., Raja, E. A., Millar, H. R., \& Bhattacharya, S. (2012). Pregnancy outcomes of women with and without a history of anorexia nervosa. Psychol Med, 42(12), 2651-2660. doi:10.1017/s0033291712000414

Easter, A., Bye, A., Taborelli, E., Corfield, F., Schmidt, U., Treasure, J., \& Micali, N. (2013). Recognising the symptoms: how common are eating disorders in pregnancy? Eur Eat Disord Rev, 21(4), 340-344. doi:10.1002/erv.2229 
Easter, A., Treasure, J., \& Micali, N. (2011). Fertility and prenatal attitudes towards pregnancy in women with eating disorders: results from the Avon Longitudinal Study of Parents and Children. BJOG: An International Journal of Obstetrics \& Gynaecology, 118(12), 1491-1498. doi:10.1111/j.1471-0528.2011.03077.x

Eddy, K. T., Dorer, D. J., Franko, D. L., Tahilani, K., Thompson-Brenner, H., \& Herzog, D. B. (2008). Diagnostic crossover in anorexia nervosa and bulimia nervosa: implications for DSM-V. Am J Psychiatry, 165(2), 245-250. doi:10.1176/appi.ajp.2007.07060951

Eik-Nes, T., Romild, U., Guzey, I., Holmen, T., Micali, N., \& Bjornelv, S. (2015). Women's weight and disordered eating in a large Norwegian community sample: the NordTrondelag Health Study (HUNT). BMJ Open, 5(10), e008125. doi:10.1136/bmjopen2015-008125

Ekéus, C., Lindberg, L., Lindblad, F., \& Hjern, A. (2006). Birth outcomes and pregnancy complications in women with a history of anorexia nervosa. BJOG: An International Journal of Obstetrics \& Gynaecology, 113(8), 925-929. doi:10.1111/j.14710528.2006.01012.X

Fairburn, C. G., \& Bohn, K. (2005). Eating disorder NOS (EDNOS): an example of the troublesome "not otherwise specified" (NOS) category in DSM-IV. Behav Res Ther, 43(6), 691-701. doi:10.1016/j.brat.2004.06.011

Field, A. E., Sonneville, K. R., Micali, N., Crosby, R. D., Swanson, S. A., Laird, N. M., . . Horton, N. J. (2012). Prospective Association of Common Eating Disorders and Adverse Outcomes. Pediatrics, 130(2), e289-e295. doi:10.1542/peds.2011-3663

Figueras, F., \& Gratacos, E. (2014). Update on the diagnosis and classification of fetal growth restriction and proposal of a stage-based management protocol. Fetal Diagnosis and Therapy, 36(2), 86-98. doi:10.1159/000357592

Franko, D. L., Blais, M. A., Becker, A. E., Delinsky, S. S., Greenwood, D. N., Flores, A. T., . .. Herzog, D. B. (2001). Pregnancy complications and neonatal outcomes in women with eating disorders. Am J Psychiatry, 158(9), 1461-1466.

Garner, D. M., \& Garfinkel, P. E. (1979). The Eating Attitudes Test: an index of the symptoms of anorexia nervosa. Psychol Med, 9(2), 273-279.

Grice, D. E., Halmi, K. A., Fichter, M. M., Strober, M., Woodside, D. B., Treasure, J. T., . . Berrettini, W. H. (2002). Evidence for a susceptibility gene for anorexia nervosa on chromosome 1. Am J Hum Genet, 70(3), 787-792. doi:10.1086/339250

Hager, R. M., Daltveit, A. K., Hofoss, D., Nilsen, S. T., Kolaas, T., Oian, P., \& Henriksen, T. (2004). Complications of cesarean deliveries: rates and risk factors. Am J Obstet Gynecol, 190(2), 428-434. doi:10.1016/j.ajog.2003.08.037

Henriksen, T. B., Wilcox, A. J., Hedegaard, M., \& Secher, N. J. (1995). Bias in studies of preterm and postterm delivery due to ultrasound assessment of gestational age. Epidemiology, 6. doi:10.1097/00001648-199509000-00012

Irgens, L. M. (2000). The Medical Birth Registry of Norway. Epidemiological research and surveillance throughout 30 years. Acta Obstet Gynecol Scand, 79(6), 435-439.

Irgens, L. M. (2002). [Medical birth registry--an essential resource in perinatal medical research]. Tidsskrift for den Norske Laegeforening, 122(26), 2546-2549.

Johansson, K., Cnattingius, S., Naslund, I., Roos, N., Trolle Lagerros, Y., Granath, F., . . Neovius, M. (2015). Outcomes of pregnancy after bariatric surgery. N Engl J Med, 372(9), 814-824. doi:10.1056/NEJMoa1405789

Kimmel, M. C., Ferguson, E. H., Zerwas, S., Bulik, C. M., \& Meltzer-Brody, S. (2016). Obstetric and gynecologic problems associated with eating disorders. Int J Eat Disord, 49(3), 260-275. doi:10.1002/eat.22483 
Koubaa, S., Hallstrom, T., Lindholm, C., \& Hirschberg, A. L. (2005). Pregnancy and neonatal outcomes in women with eating disorders. Obstet Gynecol, 105(2), 255-260. doi:10.1097/01.AOG.0000148265.90984.c3

Krokstad, S., Langhammer, A., Hveem, K., Holmen, T. L., Midthjell, K., Stene, T. R., . . . Holmen, J. (2013). Cohort Profile: the HUNT Study, Norway. Int J Epidemiol, 42(4), 968-977. doi:10.1093/ije/dys095

Lacey, J. H., \& Smith, G. (1987). Bulimia nervosa. The impact of pregnancy on mother and baby. Br J Psychiatry, 150, 777-781.

Le Grange, D., Swanson, S. A., Crow, S. J., \& Merikangas, K. R. (2012). Eating disorder not otherwise specified presentation in the US population. International Journal of Eating Disorders, n/a-n/a. doi:10.1002/eat.22006

Lemberg, R., \& Phillips, J. (1989). The impact of pregnancy on anorexia nervosa and bulimia. Inter Jour of Eat Disorder, 8(3), 285-295. doi:10.1002/1098108x(198905)8:3<285::aid-eat2260080304>3.0.co;2-p

Linna, M. S., Raevuori, A., Haukka, J., Suvisaari, J. M., Suokas, J. T., \& Gissler, M. (2014). Pregnancy, obstetric, and perinatal health outcomes in eating disorders. Am J Obstet Gynecol, 211(4), 392 e391-398. doi:10.1016/j.ajog.2014.03.067

Mackenbach, J. P., Stirbu, I., Roskam, A. J., Schaap, M. M., Menvielle, G., Leinsalu, M., \& Kunst, A. E. (2008). Socioeconomic inequalities in health in 22 European countries. $N$ Engl J Med, 358(23), 2468-2481. doi:10.1056/NEJMsa0707519

Micali, N., \& Schmidt, U. (2007). Psychiatric disorders and pregnancy. In T. S. M. S. M. V. O'Keane (Ed.), Psychiatric disorders and pregnancy (Vol. 190, pp. 125-142). Boca Raton, FL: CRC Press.

Micali, N., De Stavola, B., dos-Santos-Silva, I., Steenweg-de Graaff, J., Jansen, P. W., Jaddoe, V. W., . . Tiemeier, H. (2012). Perinatal outcomes and gestational weight gain in women with eating disorders: a population-based cohort study. BJOG, 119(12), 1493-1502. doi:10.1111/j.1471-0528.2012.03467.x

Micali, N., Dos-Santos-Silva, I., De Stavola, B., Steenweg-de Graaf, J., Jaddoe, V., Hofman, A., ... Tiemeier, H. (2013). Fertility treatment, twin births, and unplanned pregnancies in women with eating disorders: findings from a population-based birth cohort. BJOG. doi:10.1111/1471-0528.12503

Micali, N., Holliday, J., Karwautz, A., Haidvogl, M., Wagner, G., Fernandez-Aranda, F., . . . Treasure, J. L. (2007). Childhood eating and weight in eating disorders: a multi-centre European study of affected women and their unaffected sisters. Psychother Psychosom, 76(4), 234-241. doi:10.1159/000101502

Micali, N., Stemann Larsen, P., Strandberg-Larsen, K., \& Nybo Andersen, A. M. (2016). Size at birth and preterm birth in women with lifetime eating disorders: a prospective population-based study. Bjog, 123(8), 1301-1310. doi:10.1111/1471-0528.13825

Morgan, J. F., Lacey, J. H., \& Chung, E. (2006). Risk of postnatal depression, miscarriage, and preterm birth in bulimia nervosa: retrospective controlled study. Psychosomatic Medicine, 68(3), 487-492. doi:10.1097/01.psy.0000221265.43407.89

Morgan, J. F., Lacey, J. H., \& Sedgwick, P. M. (1999). Impact of pregnancy on bulimia nervosa. Br J Psychiatry, 174, 135-140.

Morrill, E. S., \& Nickols-Richardson, H. M. (2001). Bulimia Nervosa During Pregnancy: A Review. Journal of the American Dietetic Association, 101(4), 448-454.

Pasternak Y, W. A., Shoham-Vardi I, Sergienko R, Guez J, Wiznitzer A, Shalev H, Sheiner E. (2012). Obstetric and perinatal outcomes in women with eating disorders. J Womens Health (Larchmt), 21(1), 61-65. 
Pincus, H. A., Davis, W. W., \& McQueen, L. E. (1999). 'Subthreshold' mental disorders. A review and synthesis of studies on minor depression and other 'brand names'. $\mathrm{Br} \mathrm{J}$ Psychiatry, 174, 288-296.

Rappazzo, K. M., Lobdell, D. T., Messer, L. C., Poole, C., \& Daniels, J. L. (2017). Comparison of gestational dating methods and implications for exposure-outcome associations: an example with PM2.5 and preterm birth. Occup Environ Med, 74(2), 138-143. doi:10.1136/oemed-2016-103833

Reid, R. L., \& Van Vugt, D. A. (1987). Weight-related changes in reproduction function. Fertil Steril, 48(6), 905-913.

Rosenvinge, J. H., Perry, J. A., Bjørgum, L., Bergersen, T. D., Silvera, D. H., \& Holte, A. (2001). A new instrument measuring disturbed eating patterns in community populations: development and initial validation of a five-item scale (EDS-5). European Eating Disorders Review, 9(2), 123-132. doi:10.1002/erv.371

Siega-Riz, A. M., Haugen, M., Meltzer, H. M., Von Holle, A., Hamer, R., Torgersen, L., . . . Bulik, C. M. (2008). Nutrient and food group intakes of women with and without bulimia nervosa and binge eating disorder during pregnancy. Am J Clin Nutr, 87(5), 1346-1355.

Skjaerven, R., Gjessing, H. K., \& Bakketeig, L. S. (2000). Birthweight by gestational age in Norway. Acta Obstet Gynecol Scand, 79(6), 440-449.

Sollid, C. P., Wisborg, K., Hjort, J., \& Secher, N. J. (2004). Eating disorder that was diagnosed before pregnancy and pregnancy outcome. Am J Obstet Gynecol, 190(1), 206-210. doi:S0002937803009001 [pii]

. StataCorp. (2017). College Station, TX: StataCorp LLC.

Stewart, D. E., Raskin, J., Garfinkel, P. E., MacDonald, O. L., \& Robinson, G. E. (1987). Anorexia nervosa, bulimia, and pregnancy. Am J Obstet Gynecol, 157(5), 1194-1198.

Task Force on Hypertension in Pregnancy. (2013). Retrieved from https://www.acog.org/Resources-And-Publications/Task-Force-and-Work-GroupReports/Hypertension-in-Pregnancy

Tunon, K., Eik-Nes, S. H., \& Grottum, P. (1996a). A comparison between ultrasound and a reliable last menstrual period as predictors of the day of delivery in 15,000 examinations. Ultrasound Obstet Gynecol, 8. doi:10.1046/j.14690705.1996.08030178.x

Tunon, K., Eik-Nes, S. H., \& Grottum, P. (1996b). A comparison between ultrasound and a reliable last menstrual period as predictors of the day of delivery in 15,000 examinations. Ultrasound Obstet Gynecol, 8(3), 178-185. doi:10.1046/j.14690705.1996.08030178.x

Watson, H. J., Von Holle, A., Hamer, R. M., Knoph Berg, C., Torgersen, L., Magnus, P., . . Bulik, C. M. (2013). Remission, continuation and incidence of eating disorders during early pregnancy: a validation study in a population-based birth cohort. Psychol Med, 43(8), 1723-1734. doi:10.1017/s0033291712002516

WHO. (1992). The ICD-10 classification of mental and behavioural disorders: clinical descriptions and guidelines. Geneva: World Health Organization.

WHO. (2006). Neonatal and perinatal mortality : country, regional and global estimates. Retrieved from Geneva, Switzerland: 


\section{Tables}

Table 1. ED behaviors across ED diagnoses

\begin{tabular}{l|ccc}
\hline \hline Lifetime Eating Disorders & \multicolumn{3}{|c}{ Eating Disorder behaviors } \\
\hline \hline & Restriction & Purging & Total \\
\cline { 2 - 4 } Broad anorexia nervosa & 100 & 144 & 242 \\
Broad bulimia nervosa & 0 & 226 & 226 \\
EDNOS \& DE & 24 & 38 & 62 \\
\hline Total & 124 & 381 & 532 \\
\hline \hline
\end{tabular}

Table 2. General descriptive statistics of the cohorts

\begin{tabular}{|c|c|c|c|c|c|}
\hline & & Referent cohort & Anorexia nervosa & Bulimia nervosa & $\begin{array}{c}\text { EDNOS \& } \\
\text { subthreshold ED }\end{array}$ \\
\hline \multirow[t]{2}{*}{ Maternal age } & $\overline{\mathrm{N}}$ & 43651 & 244 & 226 & 62 \\
\hline & Mean (SD) & $27.1(5.3)$ & $26.7(4.9)$ & $26.6(5.0)$ & $25.8(5.0)$ \\
\hline Year of delivery & $\mathrm{N}$ & 43651 & 244 & 226 & 62 \\
\hline 1967-1989 & $\mathrm{N}(\%)$ & $26301(60.3)$ & $30(12.3)$ & $37(16.4)$ & $8(12.9)$ \\
\hline $1990-2015$ & $\mathrm{~N}(\%)$ & $17350(39.8)$ & $214(87.7)$ & $189(83.6)$ & $54(87.1)$ \\
\hline Marital status & $\mathrm{N}$ & 43651 & 244 & 226 & 62 \\
\hline Married, cohabitant, partner & $\mathrm{N}(\%)$ & $39079(89.53)$ & $209(85.7)$ & $189(83.6)$ & $45(72.6)$ \\
\hline Parity & $\mathrm{N}$ & 43651 & 244 & 226 & 62 \\
\hline 1 & $\mathrm{~N}(\%)$ & $16021(36.70)$ & $127(52.1)$ & $110(48.7)$ & $29(46.8)$ \\
\hline 2 & $\mathrm{~N}(\%)$ & $15281(35.03)$ & $76(31.2)$ & $75(33.2)$ & $21(33.9)$ \\
\hline 3 or more & $\mathrm{N}(\%)$ & $12341(28.27)$ & $41(16.8)$ & $41(18.1)$ & $12(19.4)$ \\
\hline Smoking during pregnancy & $\mathrm{N}$ & 8054 & 157 & 121 & 43 \\
\hline Smoking & $\mathrm{N}(\%)$ & $1016(12.6)$ & $26(16.6)$ & $12(9.9)$ & $9(20.9)$ \\
\hline \multirow[t]{2}{*}{ Prepregnancy BMI $\left(\mathrm{kg} / \mathrm{m}^{2}\right)$} & $\mathrm{N}$ & 972 & 43 & 49 & 11 \\
\hline & Mean (SD) & $25.0(4.4)$ & $22.2(3.1)$ & $23.2(3.5)$ & $27.2(9.2)$ \\
\hline
\end{tabular}


Table 3. Results from the GEE multivariable logistic regression models showing associations between different types of ED and obstetric outcomes

\begin{tabular}{|c|c|c|c|c|}
\hline Obstetric outcomes & Referent cohort & $\begin{array}{c}\text { Anorexia } \\
\text { nervosa }\end{array}$ & $\begin{array}{l}\text { Bulimia } \\
\text { nervosa }\end{array}$ & $\begin{array}{c}\text { EDNOS \& } \\
\text { sub-threshold } \\
\text { ED }\end{array}$ \\
\hline & OR $(95 \% \mathrm{CI})$ & OR $(95 \% \mathrm{CI})$ & OR $(95 \% \mathrm{CI})$ & OR $(95 \% \mathrm{CI})$ \\
\hline \multicolumn{5}{|l|}{$\begin{array}{l}\text { Small for } \\
\text { gestational age }\end{array}$} \\
\hline Cases $/ \mathrm{n}$ & $940 / 43651$ & $11 / 244$ & $4 / 226$ & $2 / 62$ \\
\hline Model 1 & Referent & $2.2(1.2-4.1)^{*}$ & $1.0(0.3-2.9)$ & $1.8(0.4-8.7)$ \\
\hline Model 2 & Referent & $2.2(1.2-4.1)^{*}$ & $0.8(0.3-2.3)$ & $1.3(0.3-6.2)$ \\
\hline Model 3 & Referent & $2.7(1.4-5.1)^{*}$ & $1.0(0.3-2.8)$ & $1.7(0.4-8.2)$ \\
\hline \multicolumn{5}{|l|}{$\begin{array}{l}\text { Large for } \\
\text { gestational age }\end{array}$} \\
\hline Cases $/ \mathrm{n}$ & $3774 / 43651$ & $14 / 244$ & $18 / 226$ & $6 / 62$ \\
\hline Model 1 & Referent & $0.7(0.4-1.2)$ & $1.0(0.6-1.6)$ & $1.3(0.6-3.1)$ \\
\hline Model 2 & Referent & $0.7(0.4-1.2)$ & $1.0(0.6-1.6)$ & $1.3(0.5-3.1)$ \\
\hline Model 3 & Referent & $0.6(0.4-1.1)$ & $0.9(0.5-1.5)$ & $1.2(0.5-2.8)$ \\
\hline \multicolumn{5}{|l|}{ Preeclampsia } \\
\hline Cases $/ \mathrm{n}$ & $1230 / 43651$ & $5 / 242$ & $12 / 226$ & $14 / 62$ \\
\hline Model 1 & Referent & $0.7(0.3-1.7)$ & $1.8(1.0-3.5)$ & $2.4(0.8-7.0)$ \\
\hline Model 2 & Referent & $0.6(0.2-1.7)$ & $1.8(1.0-3.5)$ & $2.3(0.8-6.9)$ \\
\hline Model 3 & Referent & $0.5(0.2-1.4)$ & $1.6(0.9-3.0)$ & $2.1(0.8-6.2)$ \\
\hline \multicolumn{5}{|l|}{ Preterm birth } \\
\hline Cases $/ \mathrm{n}$ & $1913 / 41575$ & $11 / 231$ & $12 / 214$ & $6 / 59$ \\
\hline Model 1 & Referent & $1.0(0.5-2.0)$ & $1.3(0.7-2.4)$ & $2.4(1.0-5.9)$ \\
\hline Model 2 & Referent & $1.0(0.5-2.0)$ & $1.3(0.7-2.4)$ & $2.3(0.9-5.8)$ \\
\hline Model 3 & Referent & $0.9(0.5-1.8)$ & $1.2(0.6-2.2)$ & $2.1(0.8-5.2)$ \\
\hline \multicolumn{5}{|l|}{ Caesarian section } \\
\hline Cases $/ \mathrm{n}$ & $3604 / 43651$ & $23 / 244$ & $41 / 226$ & $12 / 62$ \\
\hline Model 1 & Referent & $1.1(0.6-1.8)$ & $2.2(1.5-3.3)^{*}$ & $2.7(1.3-5.8)^{*}$ \\
\hline Model 2 & Referent & $1.1(0.7-1.9)$ & $2.4(1.6-3.6)^{*}$ & $2.9(1.3-6.1)^{*}$ \\
\hline Model 3 & Referent & $0.8(0.5-1.2)$ & $1.7(1.1-2.5)^{*}$ & $2.0(1.0-4.2)$ \\
\hline \multicolumn{5}{|l|}{ Apgar 5 minutes } \\
\hline Cases $/ \mathrm{n}$ & $681 / 29292$ & $9 / 236$ & $3 / 220$ & $4 / 57$ \\
\hline Model 1 & Referent & $1.7(0.9-3.3)$ & $0.6(0.2-1.8)$ & $3.2(1.1-8.8)^{*}$ \\
\hline Model 2 & Referent & $1.7(0.8-3.3)$ & $0.6(0.2-1.8)$ & $3.2(1.1-8.8)^{*}$ \\
\hline Model 3 & Referent & $1.6(0.8-3.2)$ & $0.6(0.2-1.8)$ & $3.2(1.1-8.9)^{*}$ \\
\hline
\end{tabular}

*Note for Table 3: Adjusted models were tested as follows. OR and 95\% CI indicate the odds of presenting adverse obstetric outcomes outcomes. Sample size varies depending on missing data on the outcome.

Model 1 is crude model

Model 2 is adjusted for maternal age

Model 3 is additionally adjusted for year of delivery, marital status and parity 
Table 4. Results from the GEE multivariable logistic regression models showing associations between lifetime history of ED behaviors and obstetric outcomes

\begin{tabular}{|c|c|c|c|}
\hline & \multirow[t]{2}{*}{ Referent cohort } & \multicolumn{2}{|c|}{ Exposed cohort } \\
\hline & & Restriction & Purging \\
\hline Obstetric outcomes & OR $(95 \%$ CI $)$ & OR $(95 \%$ CI $)$ & OR $(95 \%$ CI $)$ \\
\hline \multicolumn{4}{|l|}{$\begin{array}{c}\text { Small for } \\
\text { gestational age }\end{array}$} \\
\hline Cases/n & $940 / 43651$ & $1 / 124$ & $15 / 381$ \\
\hline Model 1 & Referent & $0.4(0.1-2.9)$ & $1.8(1.1-3.2)^{*}$ \\
\hline Model 2 & Referent & $0.4(0.1-2.9)$ & $1.8(1.0-3.1)^{*}$ \\
\hline Model 3 & Referent & $0.5(0.1-3.6)$ & $2.3(1.3-4.0)^{*}$ \\
\hline \multicolumn{4}{|l|}{$\begin{array}{l}\text { Large for } \\
\text { gestational age }\end{array}$} \\
\hline Cases/n & $3774 / 43651$ & $8 / 124$ & $28 / 381$ \\
\hline Model 1 & Referent & $0.8(0.4-1.7)$ & $0.9(0.6-1.3)$ \\
\hline Model 2 & Referent & $0.8(0.4-1.7)$ & $0.9(0.6-1.3)$ \\
\hline Model 3 & Referent & $0.8(0.4-1.7)$ & $0.9(0.6-1.3)$ \\
\hline \multicolumn{4}{|l|}{ Preeclampsia } \\
\hline Cases $/ \mathrm{n}$ & $1230 / 43651$ & $4 / 124$ & $14 / 381$ \\
\hline Model 1 & Referent & $1.2(0.4-3.4)$ & $1.2(0.6-2.2)$ \\
\hline Model 2 & Referent & $1.2(0.4-3.4)$ & $1.2(0.6-2.1)$ \\
\hline Model 3 & Referent & $1.0(0.4-2.9)$ & $1.0(0.5-1.8)$ \\
\hline \multicolumn{4}{|l|}{ Preterm birth } \\
\hline Cases/n & 1913/41575 & $9 / 120$ & $17 / 359$ \\
\hline Model 1 & Referent & $1.7(0.8-3.5)$ & $1.2(0.7-1.9)$ \\
\hline Model 2 & Referent & $1.7(0.8-3.5)$ & $1.0(0.7-1.9)$ \\
\hline Model 3 & Referent & $1.5(0.7-3.2)$ & $1.0(0.6-1.6)$ \\
\hline \multicolumn{4}{|l|}{ Caesarian section } \\
\hline Cases/n & $3604 / 43651$ & $14 / 124$ & $59 / 381$ \\
\hline Model 1 & Referent & $1.3(0.7-2.6)$ & $1.8(1.3-2.5)^{*}$ \\
\hline Model 2 & Referent & $1.4(0.7-2.7)$ & $1.9(1.4-2.7)^{*}$ \\
\hline Model 3 & Referent & $0.9(0.5-1.8)$ & $1.3(1.0-1.9)$ \\
\hline \multicolumn{4}{|l|}{ Apgar 5 minutes } \\
\hline Cases/n & $681 / 29292$ & $6 / 118$ & $9 / 369$ \\
\hline Model 1 & Referent & $2.2(1.0-5.1)$ & $1.1(0.5-2.1)$ \\
\hline Model 2 & Referent & $2.2(1.0-5.1)$ & $1.1(0.5-2.0)$ \\
\hline Model 3 & Referent & $2.2(1.0-5.1)$ & $1.0(0.5-2.0)$ \\
\hline
\end{tabular}

*Note for Table 4: Adjusted models were tested as follows. OR and 95\% CI indicate the odds of presenting adverse obstetric outcomes outcomes. Sample size varies depending on missing data on the outcome.

Model 1 is crudel

Model 2 is adjusted for maternal age

Model 3 is additionally adjusted for year of delivery, marital status and parity 


\section{Figures}

Figure 1. The final analytic study population

Figure 2. Consort diagram. Lifetime history of eating disorders 